\title{
Analysis of the radar range extension in open-ended waveguide type geometries
}

\author{
Hamid Heidar, Ahad Tavakoli ${ }^{a}$, and Abdolali Abdipour \\ Electrical Engineering Department, Amirkabir University of Technology, Tehran, \\ 15914, Iran \\ a)tavakoli@aut.ac.ir
}

\begin{abstract}
High frequency EM backscatter modeling of large waveguide type geometries is a difficult task. In analysis of radar range profile, wider bandwidths make modeling even more challenging. Here, a new dispersive scattering center model is used based on Model Based Parameter Estimation (MBPE) approach. The model is capable of representing the radar range extension according the nonlinear variations of the scattering phase. A population-based Maximum Likelihood (ML) algorithm is used to calculate the parameters. The PRONY method is used for initialization. Wideband sparse measured frequency domain samples are the inputs of the model. The results are compared with IFFT outcomes.
\end{abstract}

Keywords: waveguide, scattering center, range extension

Classification: Electromagnetic theory

\section{References}

[1] Y.-Q. Zhang and D.-B. Ge, "A unified FDTD approach for electromagnetic analysis of dispersive objects," Progress In Electromagnetics Research, PIER 96, pp. 155-172, 2009.

[2] H. T. Anastassiu, "A review of electromagnetic scattering analysis for inlets, cavities, and open ducts," IEEE Antennas Propag. Mag., vol. 45, no. 6 , Dec. 2003.

[3] M. P. Hurst and R. Mittra, "Scattering center analysis via Prony's method," IEEE Trans. Antennas Propag., vol. AP-35, pp. 986-988, Aug. 1987.

[4] L. C. Potter, D.-M. Chiang, R. Carriere, and M. J. Gerry, "A GTD-based parametric model for radar scattering," IEEE Trans. Antennas Propag., vol. 43, pp. 1058-1067, Oct. 1995.

[5] H. Heidar and A. Tavakoli, "Wideband dispersion analysis of waveguide geometries using finite sampled data," Progress In Electromagnetics Research $M$, vol. 16, pp. 235-244, 2011.

[6] D. Censor, "Broadband spatiotemporal differential-operator representations for velocity dependent scattering," Progress In Electromagnetics Research, PIER 58, pp. 51-70, 2006.

[7] R. J. Burkholder and P. H. Pathak, "Analysis of EM penetration into and scattering by electrically large open waveguide cavities using Gaussian beam shooting," Proc. IEEE, vol. 79, no. 10, Oct. 1991. 
[8] M. Pastorino, "Stochastic optimization methods applied to microwave imaging: a review," IEEE Trans. Antennas Propag., vol. 55, no. 3, March 2007.

[9] D. S. Weile and E. Michielssen, "Genetic algorithm optimization applied to electromagnetic: a review," IEEE Trans. Antennas Propag., vol. 45, no. 3, March 1997.

[10] A. W. Rihaczek and S. J. Hershkowitz, "Radar resolution and complexImage analysis," Artech House, 1996.

\section{Introduction}

Standard numerical techniques such as Finite Element Method (FEM) and Finite Difference Time Domain (FDTD) have proven to be useful in direct scattering solutions of arbitrary shaped waveguide type geometries [1]. However, they are computationally inefficient for realistic large targets due to memory limitations [2]. In inverse problems, scattering center model is a suitable technique for characterization of various features of complex targets. Most models often assume specular reflection [3] or simple diffraction mechanisms [4] for each point scatterer in a radar down range profile. In the latter, a ML algorithm is used to calculate the unknown parameters. However, these assumptions do not hold for inherently nonpoint waveguide geometries. They are extremely frequency dispersive due to wave penetration into the waveguide. Inlets, cavities and open ducts are the most troublesome structures in aerial targets. The dispersion mechanism shifts the corresponding phase centers and causes range extension i.e. a spatial error in the corresponding 1-D radar range profile. Recently, an Enhanced Dispersive Scattering Center (EDSC) model is proposed for dispersion analysis of both canonical and non-canonical waveguide type geometries [5]. In this paper, EDSC is used to quantify the waveguide range extension by analyzing the dynamic behavior of scattering phase.

\section{Formulation}

According to the geometrical theory of diffraction (GTD), the high frequency backscattered far field due to point scatterers is

$$
E_{S}(k, r)=\sum_{m=1}^{\infty} B_{m}(k, r) e^{-j 2 k r_{m}}
$$

Where $k$ is the free space wave number, $r_{m}$ is $m^{t h}$ scatterer line of sight (LOS) radar range and $B_{m}$ is the weighting coefficient. In waveguides, the phase velocity is frequency dependent and can be used to model the scattering centers [6]. In open-ended waveguides illuminated by a high frequency plane wave, the aperture field at each assumed sub-aperture has a discrete radial beam launched into the waveguide [7]. Here, such a nonpoint dispersive scattering center (DSC) is considered as an ensemble of $M$ equivalent 
point scatterers with an identical frequency dependent correction multiplicand $\xi_{m}(k)$ that is called the dispersion factor (DF). Various geometries have different DFs. Thus:

$$
B_{m}(k, r)=a_{m}(r) \xi_{m}(k)=a_{m}(r) \rho_{m}(k) e^{-j \varphi_{m}(k)}
$$

and (1) becomes

$$
E_{S}^{D S C}(k) \approx \sum_{m=1}^{M} a_{m} \rho_{m}(k) e^{-j\left(2 k r_{m}+\varphi_{m}(k)\right)}=\sum_{m=1}^{M} A_{m}(k) e^{-j \Psi_{m}(k)}
$$

where $\varphi_{m}(k)$ represents the deviation from the linear phase that is encountered in dispersive media. $\Psi_{m}(k)$ and $A_{m}(k)$ are the frequency dependent total nonlinear phase and total complex scattering amplitude respectively. For the $m^{\text {th }}$ equivalent point scattering center, a corresponding nonlinear group delay is defined as the derivative of the phase:

$$
\tau_{d m}^{n l}(k)=-\frac{1}{c} \frac{\partial \Psi_{m}(k)}{\partial k}=\tau_{d m}^{c}+\tau_{d m}^{d e v}(k) \quad(\mathrm{sec})
$$

where $\tau_{d m}^{c}=-2 r_{m} / c$ is a frequency independent term due to the radar test range and $c$ is the free space propagation velocity. The second term $\tau_{d m}^{d e v}=-\left(\partial \varphi_{m}(k) / \partial k\right) / c$ is the group delay deviation due to the scattering phase nonlinearity, which is the source of the range extension. An arbitrary open-ended curved waveguide duct is shown in Fig. 1 as an isolated DSC.

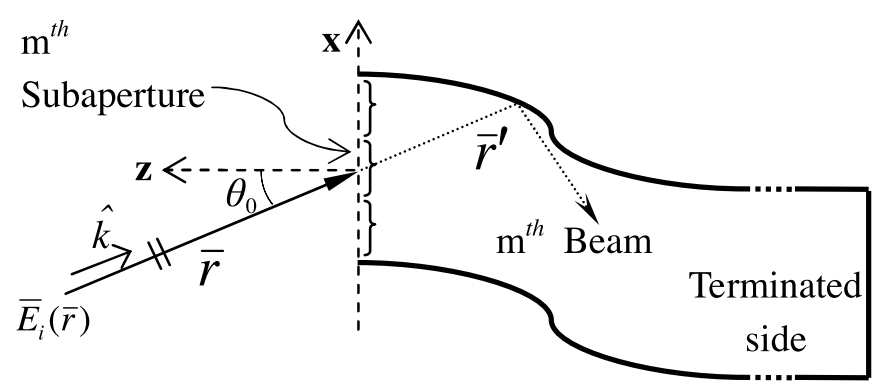

Fig. 1. Arbitrary open-ended curved waveguide duct.

A plane wave of amplitude $E_{0}$ is illuminating the open side at a small aspect angle $\theta_{0}$ permitting wave penetration into the waveguide. The radar range in non-dispersive medium (free space) and dispersive medium (waveguide) are denoted by $r$ and $r^{\prime}$ respectively. Here, the backscattered field due to the round trip propagation inside the waveguide is the dominant contributor to the total field [7]. Monostaticly, it is the main source of range extension at small aspect angles. At the aperture, it is approximated as:

$$
\hat{E}_{S}^{w g d}\left(k, r^{\prime}\right) \approx \Gamma\left(r^{\prime}\right) E_{0} e^{-2 \gamma(k) r^{\prime}}=\Gamma\left(r^{\prime}\right) E_{0} e^{-2 \alpha(k) r^{\prime}} e^{-j 2 \beta(k) r^{\prime}}
$$

where $\Gamma\left(r^{\prime}\right)$ is the waveguide reflection coefficient and $\gamma(k)$ is the complex frequency dependent propagation constant. Assuming a symmetrical crosssection, the attenuation constant $\alpha(k)$ and the phase constants $\beta(k)$ could 
be approximated by

$$
\alpha(k) \approx \frac{\left(k^{2}+2 k_{c}^{2}\right) q^{\prime}}{k \sqrt{k^{2}-k_{c}^{2}}}, \quad \beta(k) \approx \sqrt{k^{2}-k_{c}^{2}} ; \quad k>k_{c}
$$

where $k_{c}$ is the cutoff frequency and $\mathrm{q}^{\prime}$ is a conductivity related structural constant. Below cutoff frequencies, $k \leq k_{c}$, backscattering is merely from the edges and the outer surface. Now, the parametric form of (5) becomes:

$$
\hat{E}_{S}^{w g d}\left(k, r^{\prime}\right)=\hat{A}\left(r^{\prime}\right) \exp \left(-\frac{\left(k^{2}+2 k_{c}^{2}\right) q}{k \sqrt{k^{2}-k_{c}^{2}}}\right) \exp \left(-j 2 d \sqrt{k^{2}-k_{c}^{2}}\right) ; \quad k>k_{c}
$$

where $q=-2 q^{\prime} r^{\prime}$ and $\hat{A}\left(r^{\prime}\right)=\Gamma\left(r^{\prime}\right) E_{0}$ is the weighting coefficient. $d$ is called the "dispersion length" and is related to the waveguide physical length. The weighting coefficient and the phase of (7) represent the needed amplitude and phase of (2). Thus, (3) becomes the novel parametric EDSC model presented in [5]:

$$
\begin{aligned}
& E_{S}^{D S C}(k) \approx \sum_{m=1}^{M} A_{m} \exp \left(-\frac{\left(k^{2}+2 k_{c m}^{2}\right) q_{m}}{k \sqrt{k^{2}-k_{c m}^{2}}}\right) \\
& \cdot \exp \left(-j 2 k\left(r_{m}+d_{m} \sqrt{1-\left(k_{c m} / k\right)^{2}}\right)\right)
\end{aligned}
$$

The five sets of unknown parameters $\left\{A_{m}, r_{m}, q_{m}, d_{m}, k_{c m}\right\} ; m=1, \ldots, M$ should be evaluated. We face an ill-posed inverse scattering problem that requires an optimization procedure for parameters estimation [8, 9]. An approximate ML algorithm using a population-based technique is used to calculate the unknown parameters [5]. Here, the ML estimate is the parameter set that minimizes the following squared error i.e. objective function:

$$
\begin{aligned}
S\left(A, q, r, d, k_{c}\right)= & \sum_{n=1}^{L} \mid X_{n}-\sum_{m=1}^{M} A_{m} \exp \left(-\frac{\left(k_{n}^{2}+2 k_{c m}^{2}\right) q_{m}}{k_{n} \sqrt{k_{n}^{2}-k_{c m}^{2}}}\right) \\
& \left.\cdot \exp \left(-j 2 k_{n}\left(r_{m}+d_{m} \sqrt{1-\left(k_{c m} / k_{n}\right)^{2}}\right)\right)\right|^{2}
\end{aligned}
$$

In (9), $X_{n} ; n=1, \ldots, L$ are the coherent measured backscattered samples. Selection of order $M$ is highly application dependent and it cannot exceed $L / 2$. The factors to be considered include complexity of the dispersive geometry, sampling conditions (bandwidth, signal-to-noise ratio, etc.) and range resolution. The PRONY method is used for initialization of $\left\{A_{m}\right\}$ and $\left\{\mathrm{r}_{\mathrm{m}}\right\}$. The initial guess for $\left\{q_{m}\right\}$ depends on the waveguide intrinsic impedance, aperture dimensions and walls surface resistivity. The initial guess for the $\left\{d_{m}\right\}$ must be in the order of the waveguide physical length. The initial waveguide cutoff frequency $\left\{k_{c m}\right\}$ is the start of the frequency bandwidth. Convergence is examined by the Root Mean Squared (RMS) error between the original measured field data and the predicted by EDSC model. The nonlinear group delay is represented by:

$$
\tau_{d m}^{n l}(k) \approx-\frac{2}{c}\left(r_{m}+\varepsilon_{m}(k)\right) \quad(\mathrm{sec}) ; \quad \varepsilon_{m}(k)=d_{m} / \sqrt{1-\left(k_{c m} / k\right)^{2}} \quad(\mathrm{~m})
$$


Where $\varepsilon_{m}(k)$ is the frequency dependent spatial displacement introduced in (4) that is proportional to $\tau_{d m}^{d e v}$. Please note that $\tau_{d m}^{n l}(k)$ is the frequency derivative of the imaginary part of the total nonlinear phase function in (8).

\section{Results}

The linearly polarized backscattered measured data of three relatively large metallic geometries of Fig. 2 a are used in our analysis for $\theta_{0}=15^{\circ}$. The $30 \times 30 \mathrm{~cm}$ reference flat plate and the $45 \mathrm{~cm}$ long $15 \times 15 \mathrm{~cm}$ straight openended duct are measured in the same test range from 1 to $18 \mathrm{GHz}$ with $L=400$. Measurements on the $1 \mathrm{~m}$ long circular open-ended cylindrical duct of $10 \mathrm{~cm}$ diameter is performed at a different test range from 8 to $12 \mathrm{GHz}$ with only $L=100$ samples. Comparisons of the super-resolution field intensity range profiles with IFFT outcomes are depicted in Fig. 2 b, 2 c, $2 \mathrm{~d}$ with good agreement.

(a)

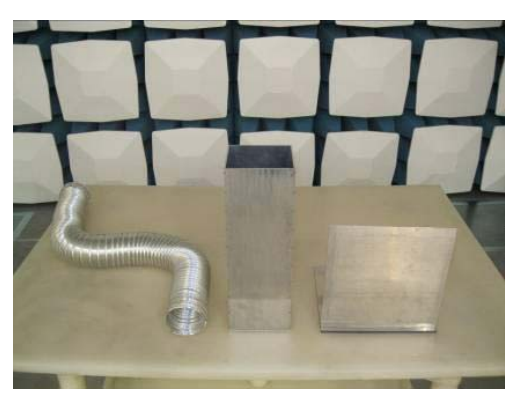

(c)

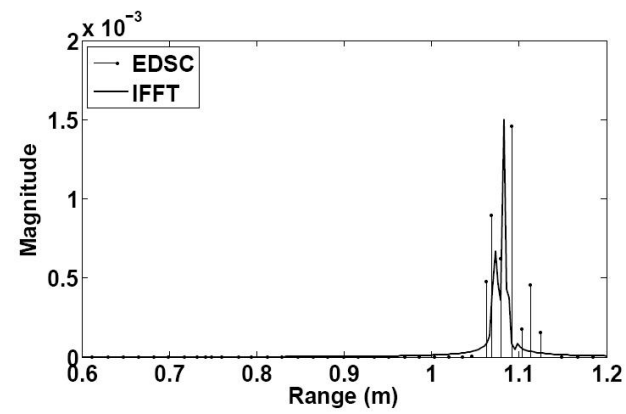

(b)

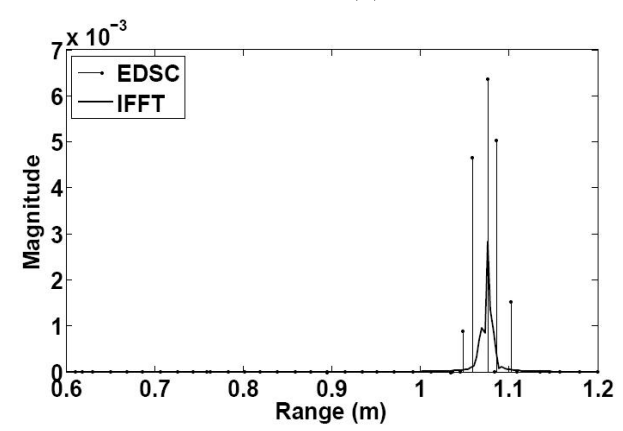

(d)

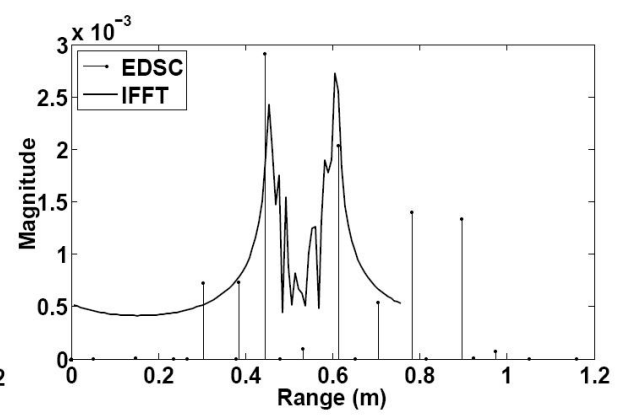

Fig. 2. (a) Metallic geometries; comparison of field intensity range profiles of (b) canonical flat plate, (c) canonical straight duct and (d) non-canonical bended duct.

In addition to intensity range profile provided by IFFT, EDSC analysis also provides the group delay curve that is used for representing the level of range extension of these targets.

The parameterized total scattering field $E_{S}^{D S C}$ is represented by (8). The unknown parameters are determined by minimization of the objective function (9). The parametric group delay (10) is derived as plotted in Fig. 3. The nonlinear scattering phase is proportional to the target location. For 
(a)

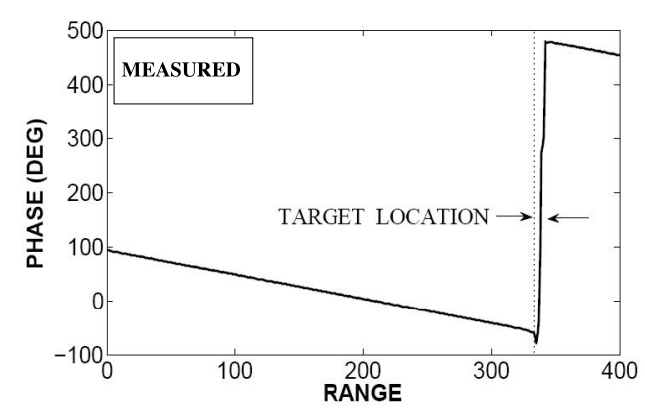

(c)

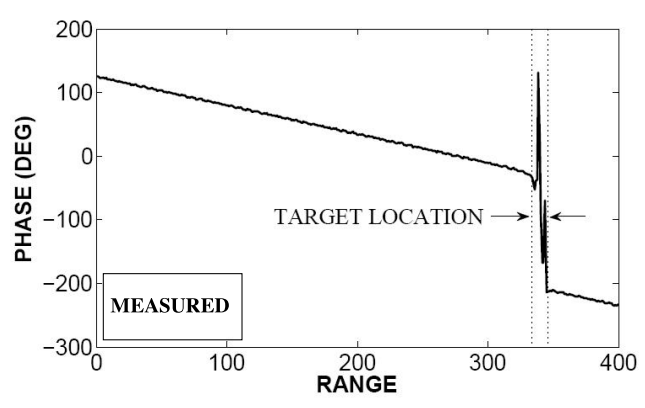

(e)

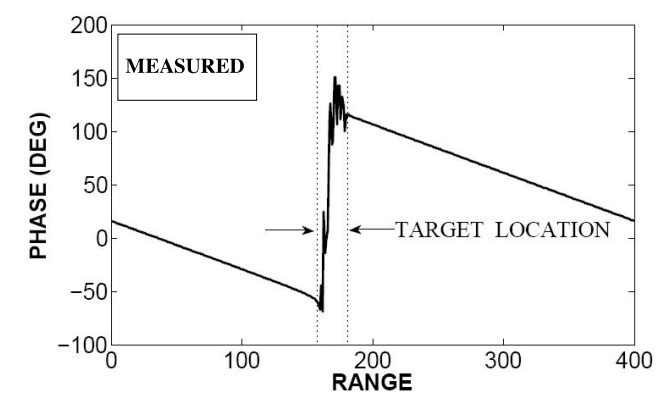

(b)

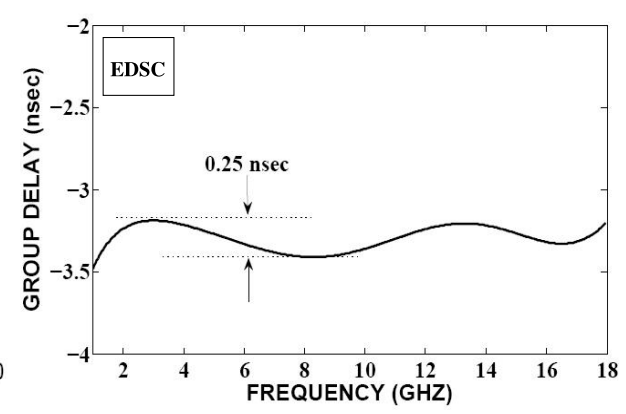

(d)

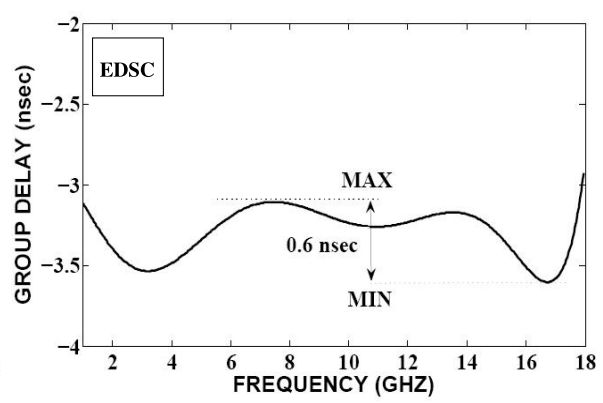

(f)

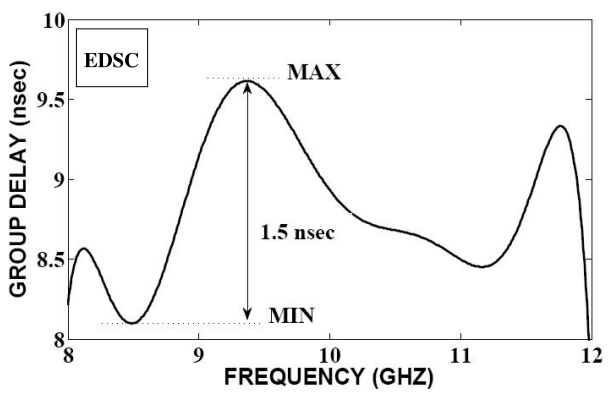

Fig. 3. Measured scattering phase versus radar range and EDSC determined group delay for $(\mathrm{a}, \mathrm{b})$ canonical flat plate, (c, d) canonical duct and (e, f) noncanonical duct.

the flat plate, the target location contains a linear sharp phase transition from the range gates of 330 to 340 (Fig. 3 a). The corresponding group delay curve with a ripple of about 0.25 nsec represents a negligible range extension (Fig. $3 \mathrm{~b}$ ). For the canonical straight duct, the target range gates from 330 to 345 show an apparent non-linear phase variation due to the dispersion as shown in Fig. $3 \mathrm{c}$. Accordingly, the group delay ripple of about $0.6 \mathrm{nsec}$ in Fig. $3 \mathrm{~d}$ represents a noticeable range extension. For the non-canonical bended duct, the nonlinear phase distortion is observed in wider range gates of 160 to 180 shown in Fig. 3 e and a larger ripple of $1.5 \mathrm{nsec}$ that is depicted in Fig. $3 \mathrm{f}$. The algorithm converged with RMS error value of $0.01,0.0031$ and 0.0044 for flat plate, canonical straight duct and non-canonical bended duct respectively. In summary, longer waveguide length leads to a higher dispersion length and range extension. According to (10), the dynamic behavior 
of the parametric group delay is appropriate for representing the range extension. Parameterization of the nonlinear variations of the scattering phase is beneficial in complex range profile analysis [10].

\section{Conclusion}

Waveguide geometries expand the radar range profiles due to dispersion. A dispersive scattering center model, EDSC is used for quantifying the range extension of such geometries. The total nonlinear scattering phase response is modeled as a combination of the linear free space LOS propagation phase and an additional nonlinear frequency dependent term. The radar range extension is modeled by the dynamic behavior of a parametric group delay quantity, which is related to the scattering phase nonlinearities. The presented complex range profile analysis includes both the scattering phase and the field intensity. Hence, it provides more information about the dispersive targets compared to IFFT that delivers only intensity range profiles. 